\title{
Toward a 3D coupled atomistic and discrete dislocation dynamics simulation: dislocation core structures and Peierls stresses with several character angles in FCC aluminum
}

Jaehyun Cho, Till Junge, Jean-François Molinari and Guillaume Anciaux ${ }^{*}$

\section{*Correspondence:}

guillaume.anciaux@epfl.ch Civil Engineering Institute, Materials Science and Engineering Institute, École Polytechnique Fédérale de Lausanne (EPFL), Station 18, $\mathrm{CH}-1015$ Lausanne, Switzerland

\begin{abstract}
Background: We present a robust method to obtain the displacement field of a dislocation core, which is one of the building blocks for the development of a direct multiscale method coupling an atomistic domain to a discrete dislocation dynamics engine in 3D (e.g. CADD3D).

Methods: The core structure is achieved by modeling of a straight dislocation with an arbitrary mixed angle using atomistic simulation. In order to validate the obtained atomistic core structures, a variational Peierls-Nabarro method is extended to include arbitrary characters.

Results: Both methods show comparable dislocation core structures for all studied angles. We provide also the Peierls stress for a wide range of character angles.

Conclusions: The obtained displacement fields for the dislocation cores were fully validated. These can consequently be employed to construct the described CADD3D coupling scheme.
\end{abstract}

Keywords: CADD3D; Mixed dislocation; Character angle; Shockley partial; Variational Peierls-Nabarro method; Peierls stress

\section{Background}

The understanding of the collective motion of line defects called dislocations is important in order to predict plastic deformations of crystalline materials. This can be studied using several computational tools, including discrete dislocation dynamics (DDD) [1-4]. In DDD, a dislocation is simply represented as a set of nodes connected by straight segments [5]. Due to this simple representation, this method can handle much larger simulation sizes (order of microns) than atomistic simulations (MD), and yields results on sample sizes which are comparable to in-situ TEM experiments [6]. DDD requires several parameters, which can be obtained from atomistic models, such as a dislocation core energy and nodal mobility law [7]. With these parameters, dislocation dynamics are reasonably well approximated. However, DDD has important limitations which require ad-hoc treatments. For instance, dislocation nucleation is only possible by inserting nucleation seeds such as frank-read sources [8-10]. On the other hand, MD describes naturally dislocation

C 2015 Cho et al. This is an Open Access article distributed under the terms of the Creative Commons Attribution License (http:// creativecommons.org/licenses/by/4.0), which permits unrestricted use, distribution, and reproduction in any medium, provided the original work is properly credited. 
nucleation as it represents dislocations explicitly as crystalline line defects, although the much larger computational cost is an important drawback. Therefore, it is attractive to combine the advantages of both approaches within a multiscale framework.

Currently, several multiscale methods exist including quasi-continuum [11], bridging domain [12] and finite element (FE) combined with atomistic modeling [13]. All these methods couple energies or displacements between two different domains: MD and FE. The authors are developing a 3D direct-coupling method between MD and DDD, which is named CADD3D ${ }^{1}$ and is an extension of the CADD2D [14] approach. In this approach, $\mathrm{MD}$ is used where dislocation nucleation are expected (and where DDD would require an ad-hoc treatment), whereas in the remaining zones standard DDD is employed. The key point of the CADD3D method is to deal with dislocations passing seamlessly between these two different domains. One notable difficulty is that a dislocation line can cross the interface between the coupled models. Then, the distinct representation of a dislocation, being either an atomistic structure or a set of DDD segments should match perfectly at the coupling interface. This can be achieved effectively by imposing respective boundary conditions to both MD and DDD regions. In this paper, we focus on the MD boundary conditions imposed by DDD: an adequate position and displacement field matching the MD core structures has to be imposed to all boundary atoms within the influence region of dislocations. The details of this approach, commonly called the core template technique will be described in Section "Coupled atomistic and discrete dislocations in 3D: core template". Because the dislocation structure is varying with its character angle ${ }^{2}$, the core template of an arbitrary mixed angle has to be built.

We explain in Section "Coupled atomistic and discrete dislocations in 3D: core template" the importance of the core template boundary condition for CADD3D to successfully build the multiscale framework. In order to obtain this core template, we provide comprehensive modeling techniques to create a dislocation with arbitrary character angles (Section "Method 1: Atomistic dislocation modeling"). We also provide an extended variational Peierls-Nabarro (PN) method [15] to validate the obtained MD core structures (Section "Method 2: Variational Peierls-Nabarro method"). Interestingly, the extended PN method can be used to predict core structures of mixed dislocations which cannot be obtained with MD simulations due to limitation of computational cost. With the successful extension of PN method, we show favorable core structures when compared to $\mathrm{MD}$, and analyze the details of dislocation core structures of all the studied character angles (Section "Results 1: Comparison between the MD and PN models core structures"). We also measure Peierls stresses for various mixed dislocations (Section "Results 2: Peierls stresses"), and show that Peierls stresses are largely influenced by the atomistic structures of the dislocation core. Finally, the paper concludes with a discussion on future work towards a fully working implementation of CADD3D.

\section{Coupled atomistic and discrete dislocations in 3D: core template}

The coupled atomistic and discrete dislocation (CADD) method [14] is a partitioneddomain, concurrent multiscale coupling method that couples a molecular dynamics or statics subdomain to a discrete dislocation dynamics subdomain and allows dislocations to transition between them. This possibility of passing dislocations between the subdomains makes CADD the only coupling method that, in principle, 
can handle the development of large dislocation networks at scalable computational cost. ${ }^{3}$

Even though only plane-strain two-dimensional implementations of CADD exist to date, the method has been successfully used for the study of such problems as dislocation emission from crack tips, void growth or nanoindentation in quasistatic (zero temperature) problems [14], fully dynamic problems at finite temperature [16] and intermediate problems where finite temperature atomistic is coupled to quasistatic discrete dislocations [17].

The key impediment to a three-dimensional CADD method is the smooth transition of dislocations across the coupling interface. The stress-strain fields around a dislocation core can be split into a nonlinear core region, where continuum mechanics fails to provide an adequate description and a far-field region, where continuum solutions suffice, see ([5], Chapter 10) or ([18], Chapter 8). While MD simulations handle both the core and the far-field correctly, the continuum with coupled FE and DDD modeling provides accurate strains - and therefore displacements- only for the far-field. As a consequence, spurious forces act on dislocation cores that approach the interface in coupled systems. When a MD dislocation comes close to the interface (see Fig. 1), its (normally) nonlinear core is intersected by the coupling interface. The continuum part of the core (dark gray area in Fig. 1) is constrained to continuum elasticity instead of reacting highly nonlinearly and therefore exhibits exaggerated stresses, leading to a repulsive spurious force on the dislocation.

In order to avoid or at least reduce such spurious forces, we propose the use of socalled core templates. The main idea of the dislocation core template is to correct the linear displacement field in the core region by a precomputed empirical and nonlinear displacement field. The exact core template $\Delta \tilde{\vec{u}}_{\mathrm{c}}\left(\vec{x}_{0}\right)$ for an atom with original position $\vec{x}_{0}$

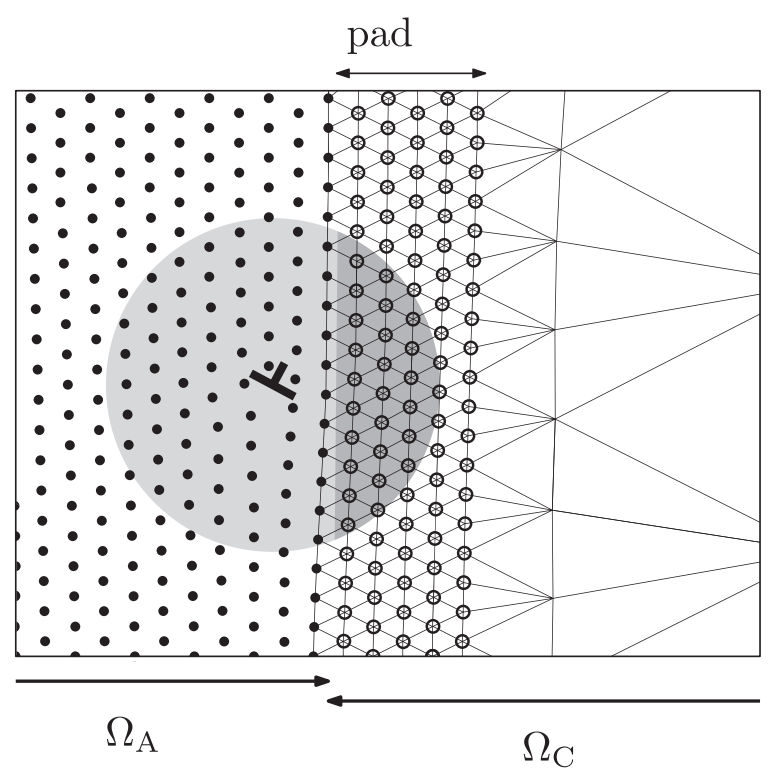

Fig. 1 Dislocation in the vicinity of the interface: the nonlinear core region (schematically represented by the gray area) interacts with the continuum across the interface. The continuum part of the core (dark gray) is linear elastic while the atomistic part is nonlinear, leading to spurious forces. $\Omega_{\mathrm{A}}$ and $\Omega_{\mathrm{C}}$ denote the atomistic and continuum subdomains. Figure from [19] 
is the error of the plastic contribution $\tilde{\vec{u}}\left(\vec{x}_{0}\right)$ of a dislocation network in a system without elastic strain $\hat{\vec{u}}\left(\vec{x}_{0}\right)=\overrightarrow{0}$

$$
\begin{aligned}
& \Delta \tilde{\vec{u}}_{\mathrm{c}}\left(\vec{x}_{0}\right)=\vec{u}_{\mathrm{A}}\left(\vec{x}_{0}\right)-\tilde{\vec{u}}\left(\vec{x}_{0}\right), \\
& =\vec{u}_{\mathrm{A}}\left(\vec{x}_{0}\right)-\sum_{j} \tilde{\vec{u}}_{j}\left(\vec{x}_{0}\right),
\end{aligned}
$$

where $\vec{u}_{\mathrm{A}}\left(\vec{x}_{0}\right)$ is the exact atomistic solution and $\tilde{\vec{u}}_{j}\left(\vec{x}_{0}\right)$ is the contribution of the $j$-th discrete dislocation segment. The template is a corrective field for the DDD solution. Assuming that the atomistic solution can be split into individual contributions from the discrete dislocation segments as well

$$
\vec{u}_{\mathrm{A}}\left(\vec{x}_{0}\right)=\sum_{j} \vec{u}_{\mathrm{A}, j}\left(\vec{x}_{0}\right)
$$

the template can be expressed in terms of such contributions

$$
\Delta \tilde{\vec{u}}_{\mathrm{c}}\left(\vec{x}_{0}\right)=\sum_{j} \Delta \tilde{\overrightarrow{\tilde{u}}}_{\mathrm{c}, j}\left(\vec{x}_{0}\right)=\sum_{j}\left[\vec{u}_{\mathrm{A}, j}\left(\vec{x}_{0}\right)-\tilde{\vec{u}}_{j}\left(\vec{x}_{0}\right)\right] .
$$

Far from any dislocation core, non-linear effects become negligible, and the small-strain linear elasticity solution $\tilde{\vec{u}}$ converges with the atomic solution $\vec{u}_{\mathrm{A}}$.

Figure 2 illustrates the application of the template: a dislocation line intersects the interface. Without a template, pad atoms are regular points in a DDD domain and their displacements are given by the superposition of the elastic solution $\hat{\vec{u}}$ and the solution due to all dislocation segments $\tilde{\vec{u}}$, see [14]. In order to minimise spurious forces, we distinguish between pad atoms inside the nonlinear dislocation core (gray cylinder of radius $r_{\mathrm{c}}$ in the figure) and pad atoms in the linear elastic region.

The template correction field $\Delta \tilde{\vec{u}}_{\mathrm{c}}$ is added to the pad atoms within the core, suppressing spurious forces. A more detailed description of the template can be found in [19]. Two limitations of the template approach are worth mentioning:

- Dislocation spacing: Implicit in the template definition (1) is the assumption that dislocations lines in the pad are spaced far from one another, i.e. no two dislocation

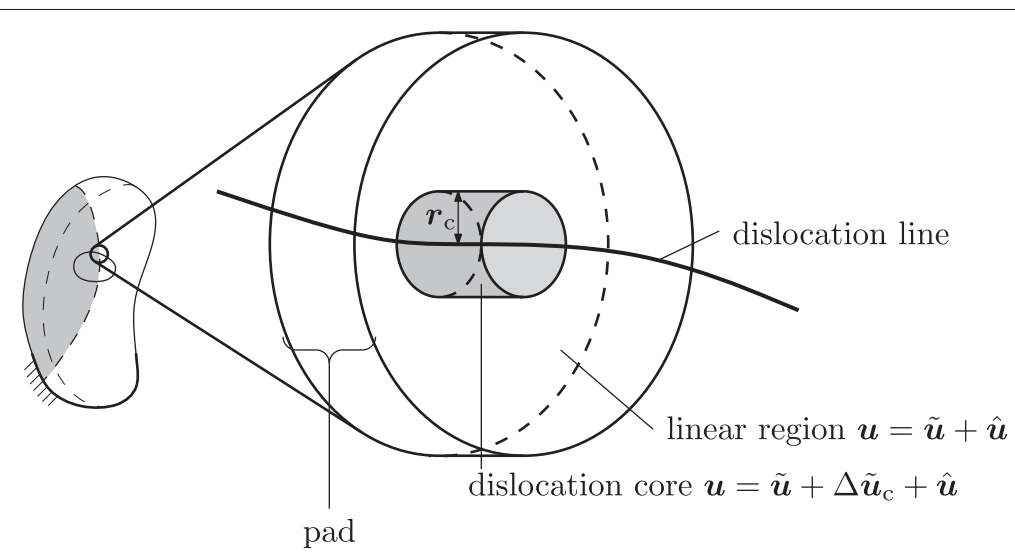

Fig. 2 Schematic of dislocation core in the pad. For most of the pad, the liner elastic DDD displacement solution is adequate, in the vicinity of the dislocation line, however, adding the core template $\Delta \tilde{\vec{u}}_{c}$ is required to minimize spurious forces. Figure from [19] 
cores overlap. If this assumption is violated and two dislocation lines approach one another, there will be spurious forces acting on them in the pad.

- Accuracy and simplicity: The template is only useful if it can be precomputed and looked up during the simulation. This means that the displacement field for dislocation cores with arbitrary character angle and curvature needs to be accurately interpolatable from a finite number of precomputed dislocation cores.

In this paper, we focus on the details of dislocation core structures with respect to the character angle in order to build valid core templates. Consequently, this study starts with a rigorous atomistic modeling of arbitrary mixed dislocations as explained in the following section.

\section{Method 1: Atomistic dislocation modeling}

A straight single dislocation can be constructed as shown in Fig. 3. A straight single dislocation is inserted in the simulation box having periodic boundary conditions (PBC) both in the dislocation gliding $(z)$ and line $(x)$ directions. As shown in Fig. 3(b), by setting the character angle $\theta$ as an arbitrary value, we can model several mixed dislocations. For an edge (respectively screw) dislocation, the dislocation line direction $\vec{d}$ is defined as perpendicular (respectively parallel) to the slip direction (Burgers vector $\vec{b}$ ) on the same slip plane. The edge $\left(90^{\circ}\right)$ and screw $\left(180^{\circ}\right)$ dislocations in FCC are modeled by choosing the dislocation line direction as $\vec{d}=[11 \overline{2}]$ for edge and $\vec{d}=[1 \overline{1} 0]$ for screw with the same Burgers vector $\vec{b}=\frac{1}{2}[1 \overline{1} 0]$. Therefore, in order to create intermediate angle dislocations $\left(90^{\circ} \leq \theta \leq 180^{\circ}\right)$, one needs to choose the dislocation line direction $\vec{d}$ accordingly. We explain the detailed procedure to find a line direction $\vec{d}$ for a specific angle dislocation as shown in Fig. 4.

The empty/full circles in Fig. 4(a) are atomistic positions in the slip plane (111). The full circles represent atoms contained in a chosen periodic unit cell, and its replications in $x$ and $z$ directions generates the other atoms as empty circles. We select one atom in the unit cell as a reference point $O$. Then, one of the neighboring atoms of $O$ indicated by the orange color arrow is chosen as shown in Fig. 4(a), which can be used to construct the direction $z^{\prime}=[3 \overline{1} \overline{2}]$ by connecting it to $O$. The cross product of the $y$ [111] and $z^{\prime}[3 \overline{1} \overline{2}]$ directions gives the remaining lattice coordinate $x^{\prime}[\overline{1} 5 \overline{4}]$ indicated by another orange arrow. Atomistic positions constructed using the new lattice coordinate system $\left(x^{\prime}, y\right.$ and $\left.z^{\prime}\right)$ are shown in Fig. 4(b). By choosing the dislocation line in $x^{\prime}$ direction with

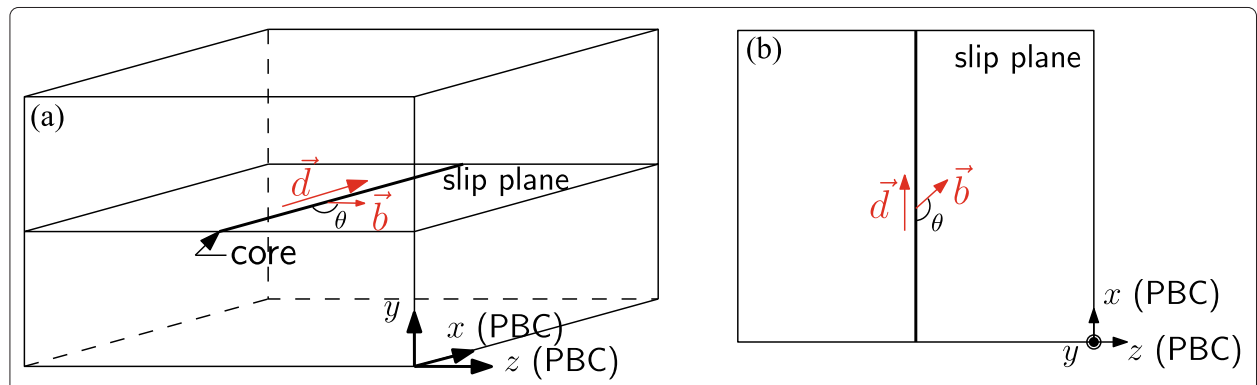

Fig. 3 (a) Schematic modeling of a straight dislocation with arbitrary character angle. (b) Top view of the slip plane. Lattice coordinate of $y$ axis is [111], while the coordinates of $x$ and $z$ axes vary with the character angle $\theta$ as shown in Table 1 


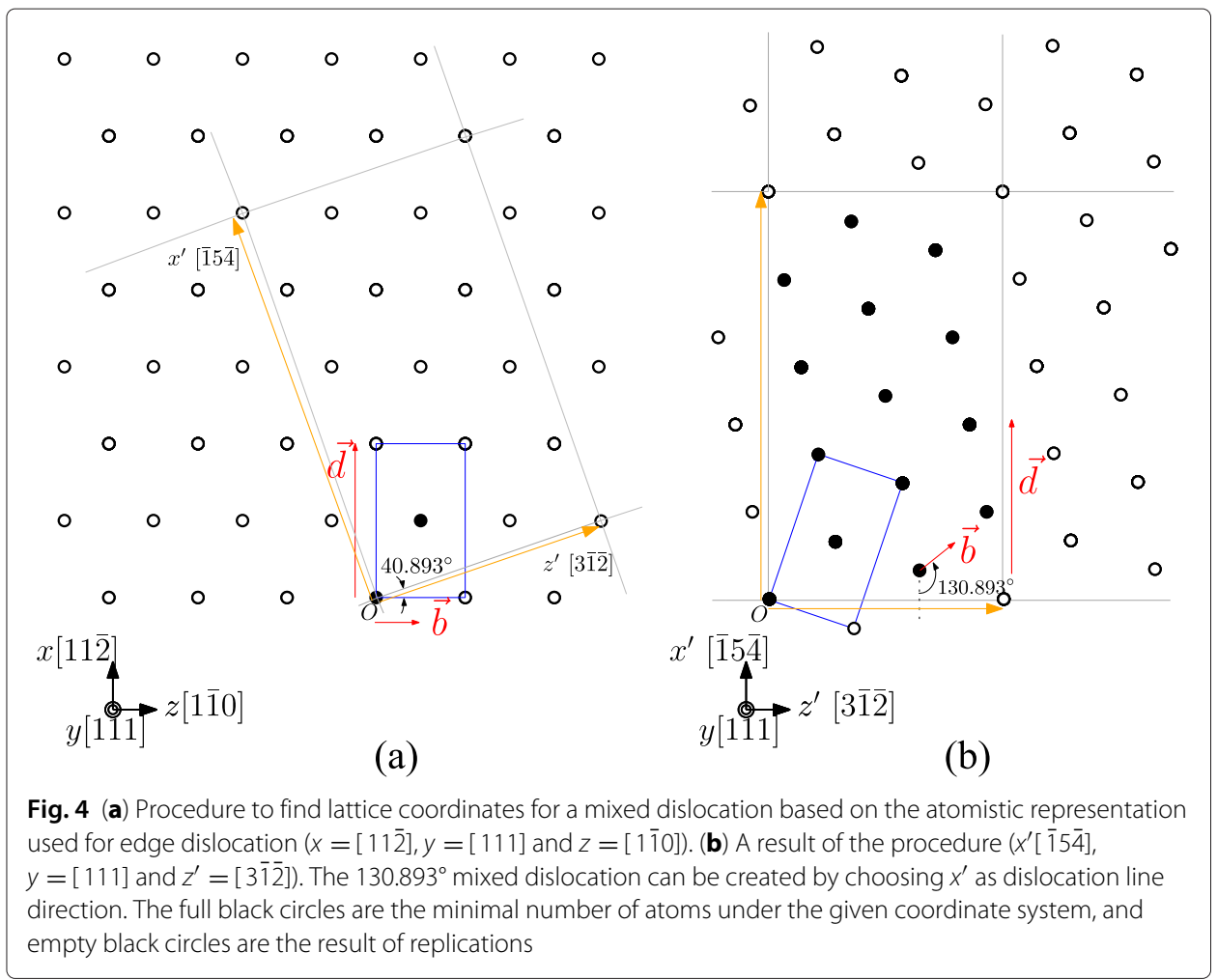

Burgers vector $\left(\vec{b}=\frac{1}{2}[110]\right)$, a mixed $\left(130.893^{\circ}\right)$ dislocation can be modeled. Other neighboring atoms, defining other $z^{\prime}$ axes, provide lattice coordinates and associated angles for other mixed dislocations. In this study, we have chosen eight representative cases which are presented in Table 1. It shows that the sizes of minimum periodic unit cells vary with the character angles. For example, the $90^{\circ}$ (edge) dislocation has the periodic unit cell with dimensions $D_{X}=\sqrt{6} a, D_{Y}=\sqrt{3} a$ and $D_{Z}=\sqrt{2} a$, while the size of the unit cell of the $130.893^{\circ}$ (mixed) dislocation is $D_{X}=\sqrt{42} a, D_{Y}=\sqrt{3} a$ and $D_{Z}=\sqrt{14} a$ with $a=4.056 \AA$ (the lattice constant). Therefore, the number of replicas need to be carefully chosen to balance evenly the number of atoms and the length of the simulation box with respect to the desired character angle.

Table 1 Selected eight angles, lattice coordinates, number of replicas and atoms of each simulation box

\begin{tabular}{cccccc}
\hline$\theta\left(^{\circ}\right)$ & $x$ & $y$ & $z$ & Replicas $(x, y, z)$ & Atoms \\
\hline 90 (edge) & {$[11 \overline{2}]$} & {$[111]$} & {$[1 \overline{1} 0]$} & $(6,10,60)$ & 86040 \\
109.107 & {$[14 \overline{5}]$} & {$[111]$} & {$[3 \overline{2} \overline{1}]$} & $(2,10,20)$ & 66900 \\
120 & {$[01 \overline{1}]$} & {$[111]$} & {$[2 \overline{1} \overline{1}]$} & $(10,10,30)$ & 71700 \\
130.893 & {$[\overline{1} 5 \overline{4}]$} & {$[111]$} & {$[3 \overline{1} \overline{2}]$} & $(2,10,20)$ & 66960 \\
139.107 & {$[\overline{1} 3 \overline{2}]$} & {$[111]$} & {$[5 \overline{1} \overline{4}]$} & $(4,10,12)$ & 80400 \\
150 & {$[\overline{1} 2 \overline{1}]$} & {$[111]$} & {$[10 \overline{1}]$} & $(6,10,60)$ & 86220 \\
160.893 & {$[\overline{2} 3 \overline{1}]$} & {$[111]$} & {$[41 \overline{5}]$} & $(4,10,12)$ & 80520 \\
180 (screw) & {$[\overline{1} 10]$} & {$[111]$} & {$[1 \overline{1} \overline{2}]$} & $(10,10,30)$ & 72000 \\
\hline
\end{tabular}


In order to insert a dislocation with a Burgers vector $\vec{b}=\frac{1}{2}[1 \overline{1} 0]$, we employ the edge and screw Volterra displacement fields $\left(u_{x}^{0}, u_{y}^{0}\right.$ and $\left.u_{z}^{0}\right)$ given by [18]

$$
\begin{aligned}
& u_{x}^{0}(z, y)=\frac{|\vec{b}|}{2 \pi} \arctan \frac{y}{z} \\
& u_{y}^{0}(z, y)=-\frac{|\vec{b}|}{2 \pi}\left(\frac{1-2 v}{4(1-v)} \ln \left(z^{2}+y^{2}\right)+\frac{z^{2}-y^{2}}{4(1-v)\left(z^{2}+y^{2}\right)}\right), \\
& u_{z}^{0}(z, y)=\frac{|\vec{b}|}{2 \pi}\left(\arctan \frac{y}{z}+\frac{z y}{2(1-v)\left(z^{2}+y^{2}\right)}\right)
\end{aligned}
$$

where $v$ is Poisson's ratio of the material. For a mixed dislocation, the displacements $\left(u_{x}^{0}\right.$, $u_{y}^{0}$ and $u_{z}^{0}$ ) are rotated by the character angle $\theta$ as

$$
\begin{aligned}
& \vec{u}_{\text {total }}(z, y)=\left(u_{x}, u_{y}, u_{z}\right) \text { with } \\
& u_{x}(z, y)=u_{x}^{0}(z, y) \cos \theta, \\
& u_{y}(z, y)=u_{y}^{0}(z, y) \sin \theta, \\
& u_{z}(z, y)=u_{z}^{0}(z, y) \sin \theta .
\end{aligned}
$$

The rotated total displacement $\vec{u}_{\text {total }}$ is imposed on the perfect crystal. Figure 5 shows the change of simulation box after imposing the displacement. The boxes before and after imposing the fields are represented by the gray dot and black solid (dashed) lines respectively. In order to create a straight boundary on the left side of the simulation box in direction $z$, image dislocations are inserted additionally on the other side of $z$ direction. The right side of the simulation box violates the PBC in direction $z$. This violation can be simply fixed by two additional geometric treatments.

First, the step created by the edge displacement fields $\left(u_{y}\right.$ and $u_{z}$ ) can be avoided by removing the extra plane (a slab of length $\vec{b} \sin \theta$ ). Second, as seen in Fig. 5(b), the opposite boundaries in direction $z$ mismatch each other by half of the screw Burgers vector $\vec{b} / 2 \cos \theta$. The dashed black lines are the boundaries of the bottom surface. This mismatch created by the screw displacement field $\left(u_{z}\right)$ is fixed by tilting the simulation box with respect to the $y$ axis in the $x$ direction by half of the corresponding screw component $-\vec{b} / 2 \cos \theta$. Consequently, the PBC in the $z$ direction is satisfied without any change of

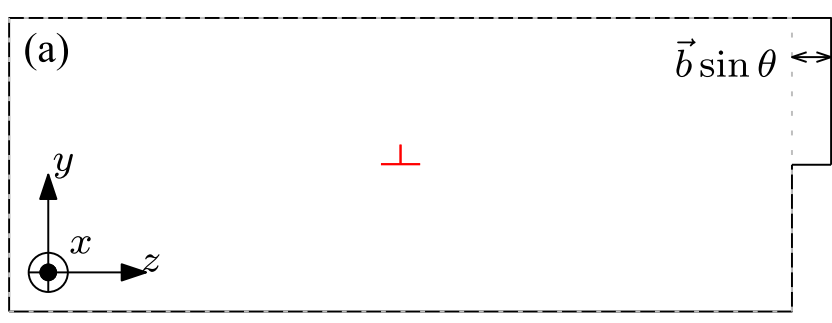

(b)

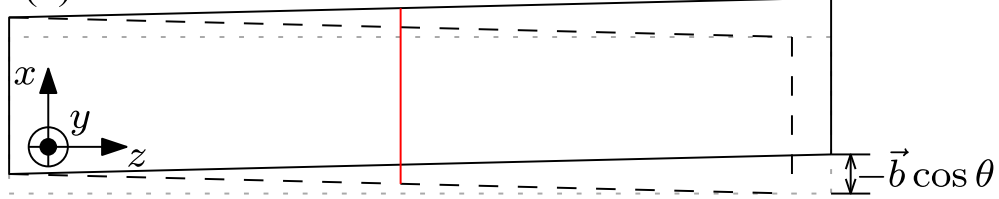

Fig. 5 Variation of the simulation box after imposing the Volterra fields. (a) front view and (b) top view. The initial box is denoted by the gray dot lines, and the box after inserting the dislocation is described with the black solid lines. The dashed black lines in (b) are the boundary of the bottom side in the $y$ direction 
the original elastic displacement field. Finally, the surfaces in the $y$ direction remain free boundaries.

In order to find the equilibrium state of dislocation core structures, the simulation box has to be relaxed. We choose the latest Aluminum EAM potential (Mendelev et al. [20]) to evaluate the inter-atomic forces. The comparison of this potential with other EAM ones will be described in Section "Method 2: Variational Peierls-Nabarro method". We relax by using a quenching process which is stopped when a norm of forces of all the atoms is below $10^{-10} \mathrm{eV} / \AA$.

Atomistic structures resulting from the relaxation are shown in Fig. 6 for the cases of $90^{\circ}$ and $130.893^{\circ}$ dislocations. The atoms are colored by the $y$ coordinates (normal to the slip plane). The initial dislocation line is dissociated into two partial dislocations. After the dissociation, the regions far away from the dislocation core show the black (A) $\rightarrow \rightarrow$ dark gray (B) $\rightarrow$ gray $(\mathrm{C}) \rightarrow$ white (A) color sequence while the area around the dislocation core shows the black $(\mathrm{A}) \rightarrow$ dark gray $(\mathrm{B}) \rightarrow$ white $(\mathrm{A})^{4}$ color sequence. The modified stacking sequence is known as a stacking fault between two Shockley partial dislocations [18] (indicated by the black arrows in the right side of Fig. 6). These partials have dissociated from a perfect dislocation $\left(\vec{b}=\frac{1}{2}[1 \overline{1} 0]=\vec{b}_{1}+\vec{b}_{2}\right.$ with $\vec{b}_{1}=\frac{1}{6}[1 \overline{2} 1]$ and $\left.\vec{b}_{2}=\frac{1}{6}[2 \overline{1} \overline{1}]\right)$ due to the energy landscape of the FCC structure. We can see that the stacking fault width for the $130.893^{\circ}$ dislocation is smaller than for the $90^{\circ}$ (edge) dislocation. Furthermore, the two partials are symmetric in the case of the edge dislocation, but not for intermediate $\left(130.893^{\circ}\right)$ dislocations. In order to understand and validate these relaxed core structures, the variational Peierls-Nabarro model, describing dislocation core structures with a continuum representation, is used in the next section.

\section{Method 2: Variational Peierls-Nabarro method}

Continuum theory of linear elasticity provides useful analytic solutions for dislocation problems because the theory offers quite accurate dislocation structures when far from the core. However, this theory cannot be employed anymore near the dislocation center, where one finds highly distorted atomistic structures. These nonlinear atomic

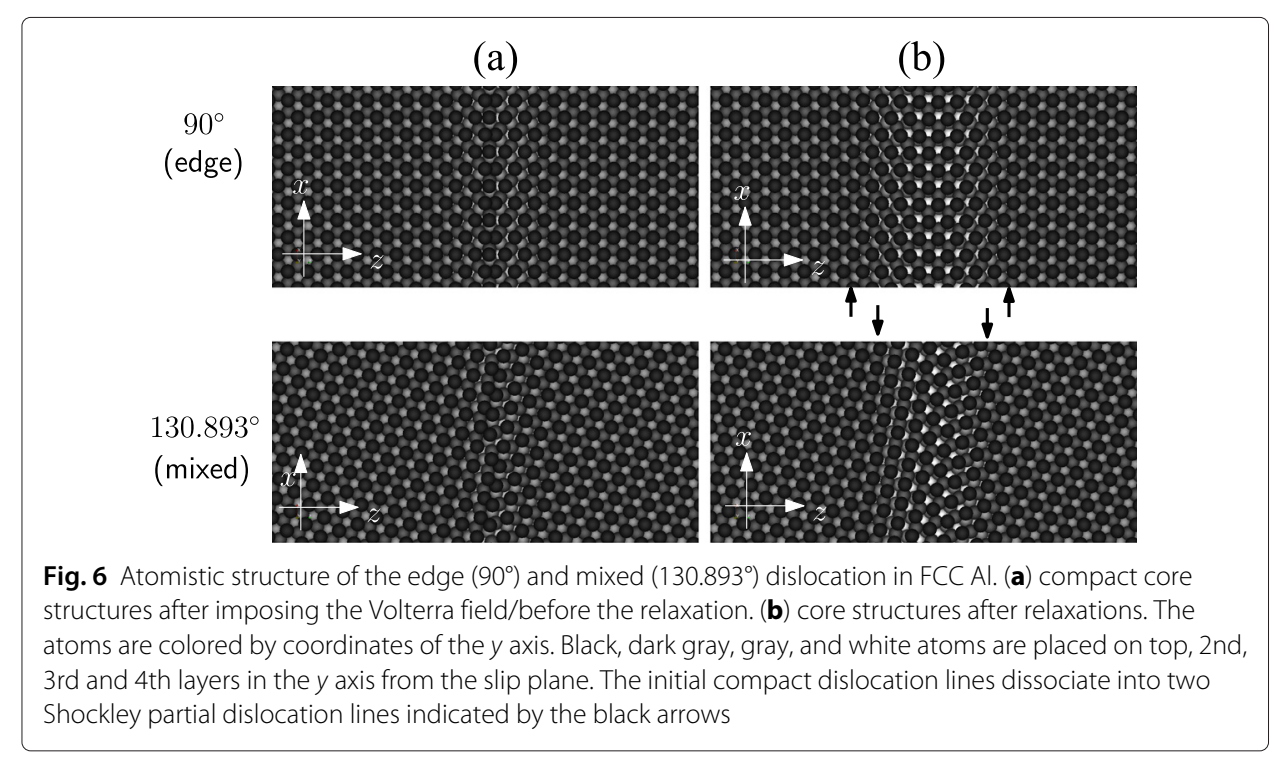


interactions can be naturally captured by an atomistic model. For these reasons, simulations combining the continuum and atomistic models can be useful not only for the far field but also for the dislocation center. One of the available tools combining the continuum and atomistic models is the variational Peierls-Nabarro (PN) method [21, 22], which generally has been used to calculate dislocation core structures by minimizing the total energy of the system. More precisely, the energy in the far field is captured by the energy formulation of continuum linear elasticity theory, while the energy in the core region is obtained by using the crystalline misfit energy and a spread displacement field on the slip plane. The total energy is the sum of both these energy formulations, and the optimal dislocation core structures are found when the total energy is minimum. The PN model is known as a tool providing good descriptions of dislocation core structures in comparison with MD results [5, 15, 23-25].

The original PN formulation starts from the total energy $E_{\text {tot: }}$ :

$$
E_{\mathrm{tot}}=E_{\mathrm{el}}+E_{\mathrm{msft}}
$$

where $E_{\mathrm{el}}$ is the elastic energy, and $E_{\mathrm{msft}}$ is the misfit energy. Because the original Volterra displacement field $\vec{u}$ is singular on the dislocation core, the original energy formulation is ill-posed. Therefore $\vec{u}$ has to be spread, which can be performed in two ways. In a first approach, the displacements are divided into edge $u_{z}$ and screw $u_{x}$ components using several arc-tangent functions $[5,15,23]$. A second method called phase field (PF) method is distributing the displacement $\vec{u}$ into all possible slip directions $u_{1}=[0 \overline{1} 1], u_{2}=[10 \overline{1}]$ and $u_{3}=[\overline{1} 10]$ using scalar functions $\zeta(z)$ recording the amount of slips [24, 26-28]. In this article, we choose the first approach without comparisons between the two methods because the both methods are known to provide sufficiently good dislocation core structures confirming the obtained atomistic simulation results. The two arc-tangent functional forms for both edge $u_{z}^{0}$ and screw $u_{x}^{0}$ displacements as described in [5]:

$$
\begin{aligned}
& u_{z}^{0}=A_{1} \arctan \frac{z-z_{1}}{c_{1}}+A_{2} \arctan \frac{z-z_{2}}{c_{2}}-\frac{b}{2}, \\
& u_{x}^{0}=A_{3} \arctan \frac{z-z_{1}}{c_{3}}+A_{4} \arctan \frac{z-z_{4}}{c_{4}}
\end{aligned}
$$

where $A_{k}, c_{k}$ and $z_{k}$ are the parameters obtained through minimization. By the principle of superposition, we rotate $u_{z}^{0}$ and $u_{x}^{0}$ by the character angle $\theta$ to have slip and line directions in $z$ and $x$ axes respectively:

$$
\begin{aligned}
& u_{z}=\frac{b}{\pi}\left(u_{z}^{0} \sin \theta-u_{x}^{0} \cos \theta\right)-\frac{b}{2}, \\
& u_{x}=\frac{b}{\pi}\left(u_{x}^{0} \cos \theta+u_{z}^{0} \sin \theta\right) .
\end{aligned}
$$

With the arc-tangent shape of displacements in hand $\left(u_{z}\right.$ and $\left.u_{x}\right)$, we can construct analytically the elastic energy $E_{\mathrm{el}}$ :

$$
\begin{aligned}
E_{\mathrm{el}}= & -K_{\text {edge }} \int_{-\infty}^{\infty} \int_{-\infty}^{\infty} \frac{d u_{z}}{d z} \frac{d u_{z}}{d z^{\prime}} \ln \left|z-z^{\prime}\right| d z d z^{\prime} \\
& -K_{\text {screw }} \int_{-\infty}^{\infty} \int_{-\infty}^{\infty} \frac{d u_{x}}{d z} \frac{d u_{x}}{d z^{\prime}} \ln \left|z-z^{\prime}\right| d z d z^{\prime}
\end{aligned}
$$

where $K_{\text {edge }}=\mu /(4 \pi(1-v)), K_{\text {screw }}=\mu / 4 \pi$. 
The misfit energy $E_{\mathrm{msft}}$ can be constructed by using Equation (10) with the misfit potentials $\gamma\left(u_{z}, u_{x}\right)$ obtained from independent MD simulations. The misfit potentials are computed by constant and rigid shifting of two crystals on the glide plane (111) against each other [29]. The results of misfit potentials of Mendelev et al. [20] potential is shown in Fig. 7(a).

$$
E_{\mathrm{msft}}=-\int_{-\infty}^{\infty} \gamma\left(u_{z}(z), u_{x}(z)\right) d z
$$

For a dislocation in FCC materials, the generalized stacking fault (GSF) energy curve in $x=\langle 11 \overline{2}\rangle$ directions is important to understand dislocation slip behaviors. The GSF energy curve was first suggested by Vitek [30] to be composed of an intrinsic stacking fault energy $\gamma_{I}$ (ISF) and an unstable stacking fault energy $\gamma_{U}$ (USF) [24, 31]. The ISF energy is the local energy minimum of partial dislocations, and the USF energy is the minimum energy required in order to translate partials. The obtained GSF energy curve in $x=\langle 11 \overline{2}\rangle$ is given in Fig. 7(b) with $\gamma_{U}=243.3 \mathrm{~mJ} / \mathrm{m}^{2}$ and $\gamma_{I}=128.6 \mathrm{~mJ} / \mathrm{m}^{2}$. When compared DFT computations and Mishin \& Farkas, our selected Mendelev et al. potential provides an accurate description of $\gamma_{U}$ and $\gamma_{I}$.

After constructing the total energy formulation, one can see that the only difference between the arbitrary character angles is the amount of elastic energy $E_{\text {el }}$ given by the terms $K_{\text {edge }}$ and $K_{\text {screw }}$ (see Equation 9), which differ by $1 /(1-v)$ : the dislocations close to edge have larger elastic energies than the dislocations around screw. The total energy $E_{\text {tot }}$ is minimized numerically to get the optimal shape of the displacement fields $\vec{u}$ over the slip plane.

\section{Results 1: Comparison between the MD and PN models core structures}

By dumping the displacements of all atoms located on the upper and lower slip planes in the MD simulation, we can compare the displacements $\vec{u}=\left(u_{x}, u_{z}\right)$ with the PN results

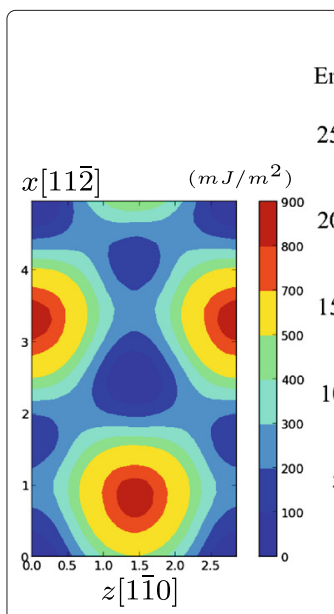

(a)

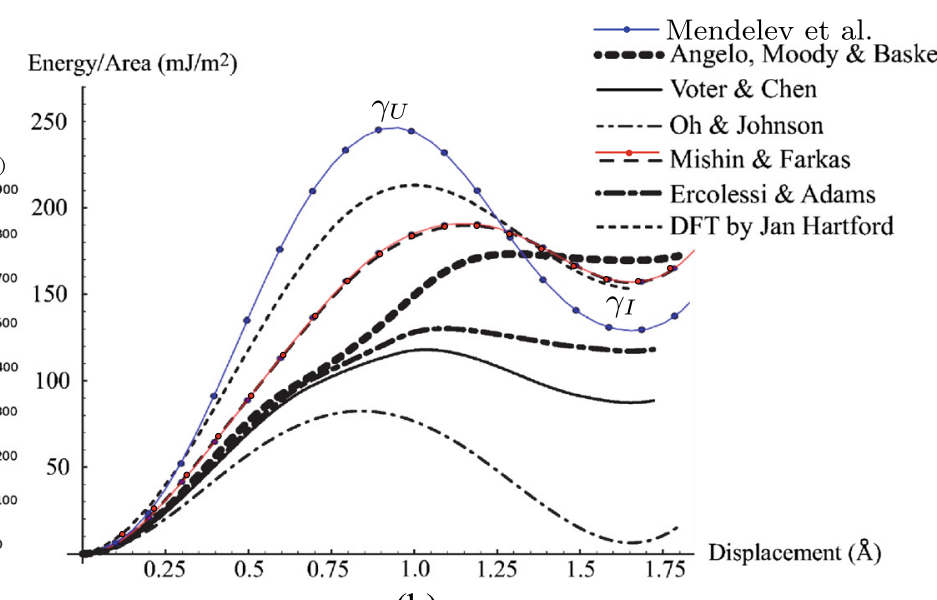

(b)

Fig. 7 (a) Periodic misfit potential in the $x$ and $z$ directions of the Mendelev et al. EAM potential [20]. (b) GSF energy curves in $\langle 112\rangle$ direction of several Al EAM potentials [32, 42-44] and DFT computations [45] (figure was taken with agreement of copyright by author $[29,46]$ and IOP publishing). The blue curve shows results obtained with the Mendelev et al. potential [20]. We reproduce one of author's results shown by the red curve with Mishin and Farkas potential [32] 
as shown in Fig. 8. The MD results are represented with markers while the PN results are described by lines. Clearly, the PN model predicts well the atomistic dislocation core structures. The edge $\left(90^{\circ}\right)$ dislocation is decomposed into the two symmetric Shockley edge and screw partial dislocations. As the angle increases, the partial displacements are no longer symmetric to each other, and finally become symmetric again at the angle of screw dislocation $\left(180^{\circ}\right)$.

The mapping of PN results onto the perfect atoms located on the slip plane is shown in Fig. 9 with blue crosses in the case of $130.893^{\circ}$ dislocations. The equilibrium state of atomistic core structures is represented in red circles. Again, both results are in good agreement in the core region.

Next, we compare the strains with respect to $z$ coordinates $(d \vec{u} / d z)$ as shown in Fig. 10 . While the strains for the $\mathrm{PN}$ results can be derived analytically since the displacements are represented by the two arc-tan functions, the strains for the MD results are obtained by computing the slope between two neighboring points without any line interpolation. We define the stacking fault width as the distance between the strain extremes, thus providing a way to measure the widths as function of the different angles (see Fig. 11). The width for the edge dislocation is $l=3.4$ Burgers, and it is in reasonable range $(2.8 \leq l \leq 13.4)$ when compared to results obtained from other simulations and potentials [32-35]. As the angle increases to screw dislocation $\left(180^{\circ}\right)$, the width decreases smoothly. It can be understood due to the decrease of the elastic energy with increasing angle.

We see that the extended PN method produces dislocation core structures, at much cheaper computational costs, that compare reasonably well with the atomistic core structures. Therefore, we can employ the PN method to predict core structures of mixed dislocations which cannot be obtained by atomistic simulations when the sizes of unit cells become large enough to exclude an atomistic calculation.

\section{Results 2: Peierls stresses}

The Peierls stress is defined as the minimum stress required to translate a dislocation at zero temperature [18], and it is known to be influenced by core structures [35-39].

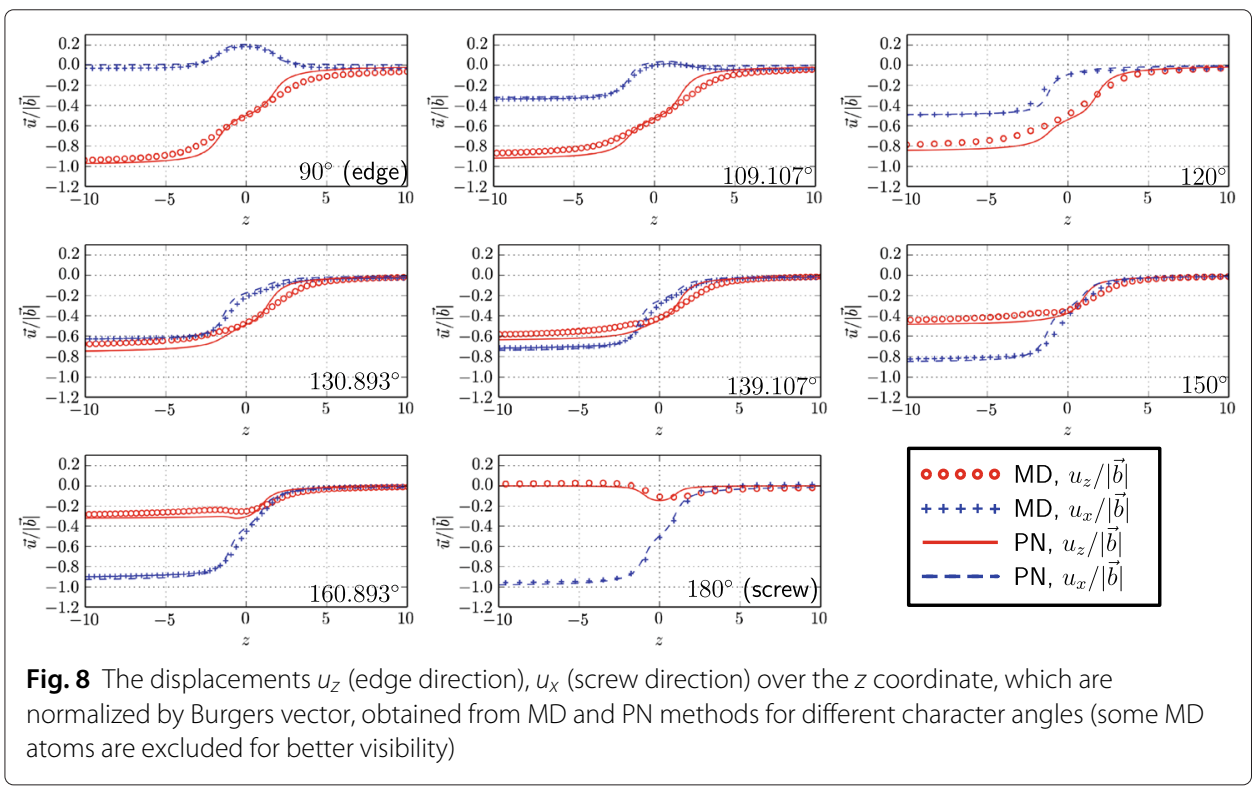




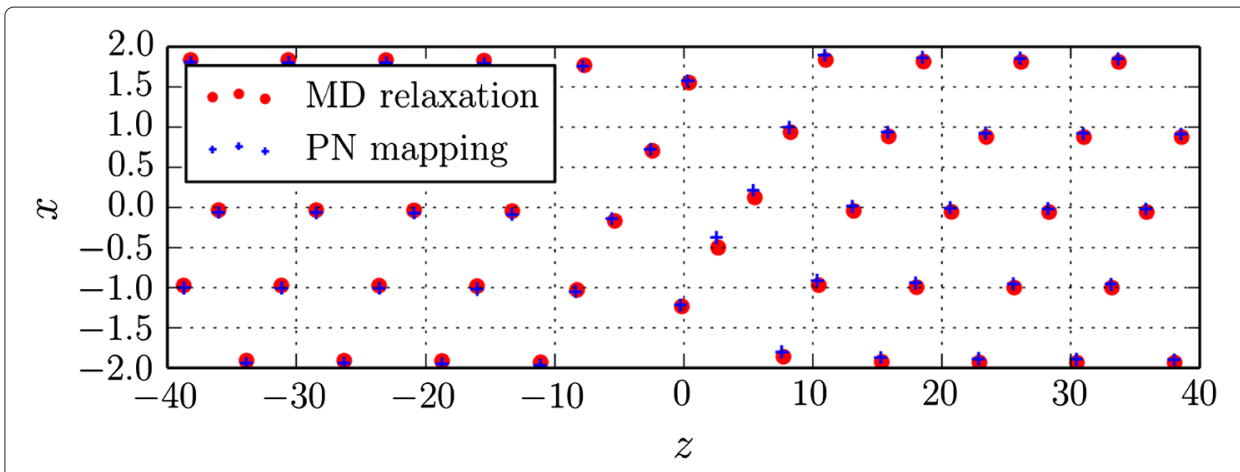

Fig. 9 Comparison between MD with PN results with atomistic representation on the slip plane $\left(130.893^{\circ}\right.$ mixed dislocation)

Because we have obtained the details of the dislocation core structures e.g., lattice orientation and stacking fault widths, the influences of the core structures on the Peierls stress can be studied in this section. Several articles $[35,37,38]$ have been published regarding the Peierls stresses of FCC Aluminum, measured using direct atomistic simulations. A wide range of results (from $1 \mathrm{MPa}$ to $13 \mathrm{MPa}$ for an edge and from $1 \mathrm{MPa}$ to $82 \mathrm{MPa}$ for a screw) is observed. Shin and Carter [38] found that a dislocation dissociated into partials has a smaller Peierls stress than a compact dislocation. Olmsted et al. [37] studied Peierls stresses for mixed dislocations $\left(120^{\circ}\right.$ and $\left.150^{\circ}\right)$ including the edge and screw character angles. They found that the screw and $120^{\circ}$ dislocations are required to overcome a higher Peierls energy barrier than the edge and $150^{\circ}$ dislocations. They argued that such results are due to the density of atoms in the motion direction (Simmons et al. [39] initially suggested this idea). To the best of our knowledge, Peierls stresses have been studied only for the four character angles $\left(90^{\circ}, 120^{\circ}, 150^{\circ}\right.$ and $\left.180^{\circ}\right)$, whereas we selected other new character angles.

In Section "Method 1: Atomistic dislocation modeling", we constructed the dislocations based on the corresponding Volterra displacement fields followed by relaxations. These
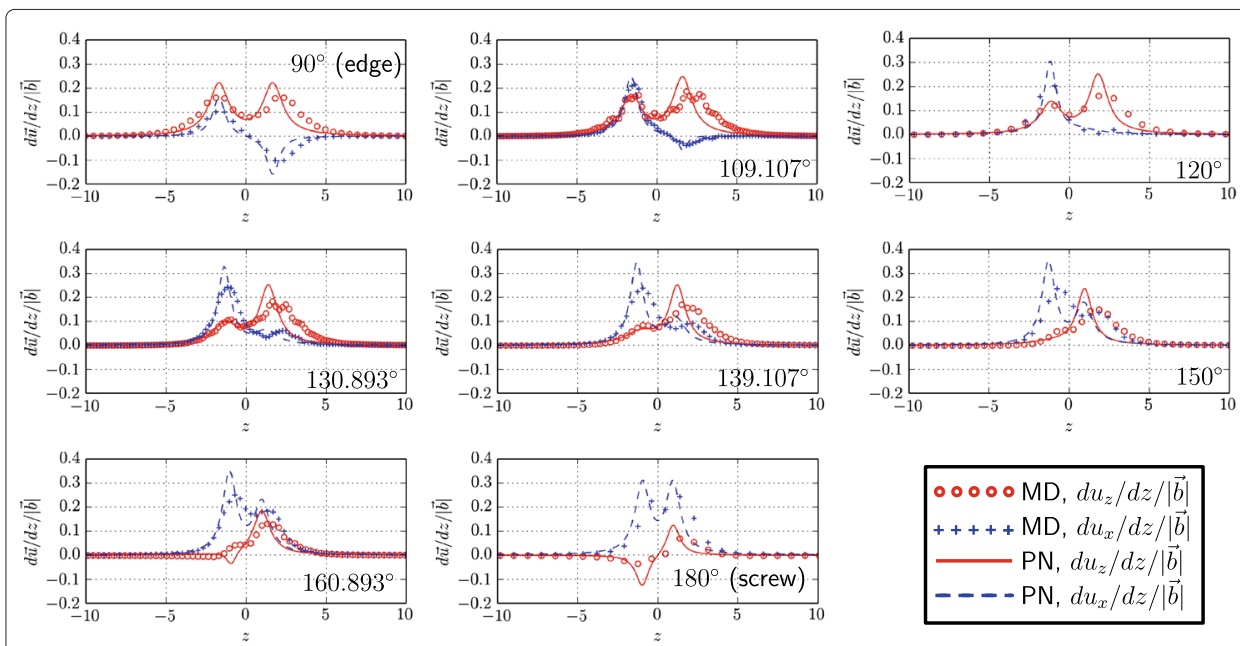

Fig. 10 The strains $\left(d u_{z} / d z\right.$ and $\left.d u_{x} / d z\right)$ over $z$ axis, which are normalized by Burgers vector, measured by $\mathrm{MD}$ and $\mathrm{PN}$ for the representative seven angles 


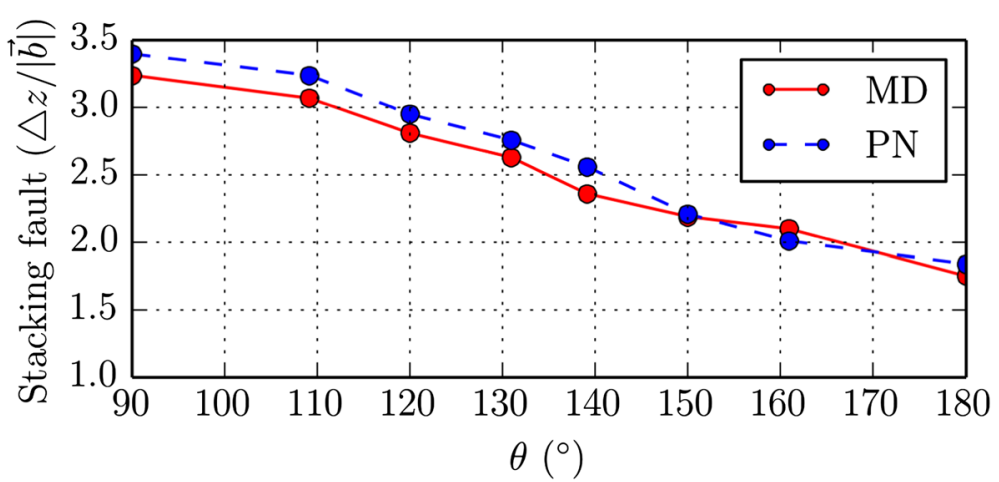

Fig. 11 Variation of stacking fault widths for several angles

relaxed fields are only valid when dislocations locate at initial positions. As the dislocations change their positions on the gliding plane, the following stresses are polluted by the artificial image stresses from the unmatched dislocation surrounding fields [37]. In order to avoid the unwanted effects from the boundaries, we imposed linear displacement fields $\left(u_{x, \text { lin }}, u_{y, \text { lin }}\right.$ and $\left.u_{z, \text { lin }}\right)$ on the atoms in the top and bottom surfaces instead of the Volterra elasticity solution. By introducing these linear fields, the boundary conditions are invariant with respect to the dislocation position. The linear displacement fields for the top and bottom surfaces are chosen as follows:

$$
\begin{aligned}
& u_{x, \operatorname{lin}}^{0}(z, y)=\frac{-\operatorname{sign}(y) b_{\text {screw }} / 2}{L_{Z}}\left(z+\frac{L_{Z}}{2}\right), \\
& u_{y, \text { lin }}^{0}(z, y)=0.0, \\
& u_{z, \text { lin }}^{0}(z, y)=\frac{-\operatorname{sign}(y) b_{\text {edge }} / 2}{L_{Z}}\left(z+\frac{L_{Z}}{2}\right) .
\end{aligned}
$$

with $L_{Z}$ the length of the simulation box in direction $z$. On the top surface, the negative Burgers $\left(-b_{\text {edge }}\right.$ and $\left.-b_{\text {screw }}\right)$ vectors are used, and the positive Burgers $\left(+b_{\text {edge }}\right.$ and $\left.+b_{\text {screw }}\right)$ are employed for the bottom surface. In order to consider the mixed character angles, the linear displacements are rotated by the given character angle $\theta$ (Equation 6). The remaining atoms are subjected to the Volterra fields, and are also rotated by $\theta$ as we described in Section "Method 1: Atomistic dislocation modeling". After imposing the various displacement fields on the atoms in each region, we relax the simulation box, and measure the Peierls stress through quasi-static loading. Specifically, we translate the atoms on the top surface along the Burgers direction in small steps, while we fix the atoms on the bottom surface. After each translation, we minimize the bulk of atoms and monitor the final energy value when the minimization finishes. Therefore, we can observe the energy variation of the system as the dislocation moves to different positions along the gliding direction.

Figure 12(a) shows the dislocation displacement $u_{\text {dis }}$ in the gliding direction $z$ with respect to the displacements of the top surface $u_{\text {top }}$ in the Burgers direction $\vec{b}$, and the corresponding potential energy variations $\Delta E_{\text {pot }}$ of the system are given in Fig. 12(b).

The dislocations slowly change their position, and suddenly jump to the next positions as the quasi-loading is incremented. The jump magnitudes of the edge dislocation are 


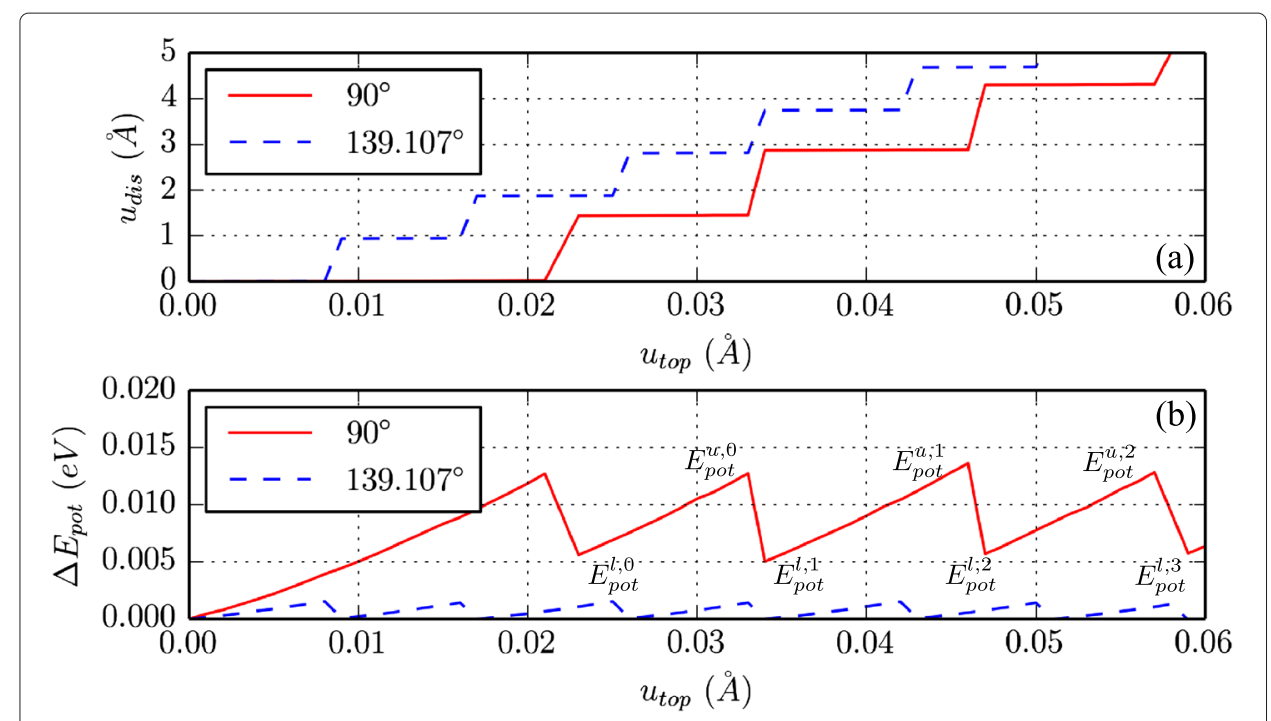

Fig. 12 a Dislocation displacements and (b) energy variations with respect to top surface displacement of the edge $\left(90^{\circ}\right)$ and mixed $\left(139.893^{\circ}\right)$ dislocation

larger than the mixed $\left(139.893^{\circ}\right)$ dislocation. Consequently, the edge dislocation requires higher energy accumulations (and reliefs) before (and after) jumps(respectively) as shown in Fig. 12(b) than the mixed dislocation. We call each energy relief and peak point $E_{\text {pot }}^{l}$ and $E_{\mathrm{pot}}^{u}$ respectively. The Peierls stress is computed as follows [40]:

$$
\sigma_{p}=\frac{\Delta E_{\mathrm{pot}}^{\max }}{b h_{z} L_{x}}=\frac{\max \left\{\left(E_{\mathrm{pot}}^{u, n-1}-E_{\mathrm{pot}}^{l, n}\right) \mid n \in[1 . . N]\right\}}{b h_{z} L_{x}}
$$

where $L_{x}$ is the length of the simulation box in dislocation line direction $x$ and $\Delta E_{\mathrm{pot}}^{\max }$ is the maximum energy release among $N$ events, $h_{z}$ is the displacement of the dislocation in $z$ direction occurred by one Burgers $\vec{b}$ slip. Cai [40] defined $h_{z}$ as the repeat distance for which the system is translation invariant, and argued that $h_{z}$ can be defined geometrically with respect to $D_{z}$ (length of a primitive unit cell in gliding direction). Since the $90^{\circ}, 120^{\circ}, 150^{\circ}$ and $180^{\circ}$ dislocations were built based on the FCC primitive unit cells, $h_{z}$ can be defined as $h_{z}=D_{z}\left(90^{\circ}\right) / 4$ in the case of FCC crystals. For example, $h_{z}$ is $|\vec{b}| / 2$ for $90^{\circ}$ and $150^{\circ}$ dislocations, and $\sqrt{3}|\vec{b}|$ for $120^{\circ}$ and $180^{\circ}$ dislocations. These distances are represented in the energy variation curves (Fig. 12) as follows. The energy of the simulation box containing a edge $\left(90^{\circ}\right)$ dislocation varies with a period $h_{z}=|\vec{b}| / 2=1.43 \AA$. Particularly, the other mixed dislocations $\left(109.107^{\circ}, 130.873^{\circ}, 139.107^{\circ}\right.$ and $\left.160.893^{\circ}\right)$ are constructed based on the periodic unit cells where several primitive unit cells exist (see Fig. 4). The repeat distances $h_{z}$ for those angles cannot be defined with respect to the primitive unit cell. Since there are 28 atoms in the given periodic unit cells meaning that 28 energy accumulation-release events happen as a dislocation moves across an entire unit cell. As shown in Fig. 12, the energy of $139.107^{\circ}$ dislocation varies periodically by $h_{z}=$ $D_{z}\left(139.107^{\circ}\right) / 28=0.935 \AA$. Using the corresponding value of $h_{z}\left(D_{z}(\theta) / 4\right.$ or $\left.D_{z}(\theta) / 28\right)$, the Peierls stresses of all the character angles can be measured with Equation 12. The measured Peierls stresses are shown in Fig. 13 with respect to the density of atoms in the gliding direction. 


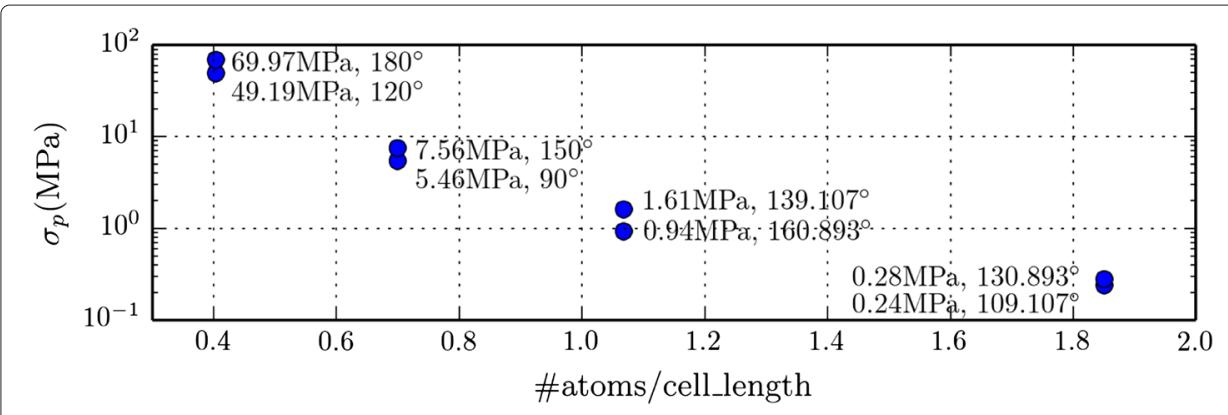

Fig. 13 Variation of Peierls stresses for the several angles with respect to the density of atoms in the gliding direction. The corresponding Peierls stress value and the character angle are denoted next to each marker

We can first compare Peierls stresses of the dislocations constructed with an equivalent lattice orientation (but with a different character angle). As shown in Table 1, the edge and $150^{\circ}, 109.109^{\circ}$ and $130.893^{\circ}, 139.109^{\circ}$ and $160.893^{\circ}, 120^{\circ}$ and screw dislocations are modeled based on the same lattice orientation. For each lattice orientation, the Peierls stress decreases low as the character angle decreases. These observations were confirmed by Simmons et al. [39], and it can be understood with the magnitudes of dislocation dissociation [38]. As seen in the variation of stacking fault widths in Fig. 11, the dislocations are dissociated into larger stacking fault widths when their character angles become small. Consequently, the Peierls stress decreases as the character angle reduces. In the case of $139.109^{\circ}$ and $160.893^{\circ}$ dislocations, the above argument is not valid, and we have not found any reasons for the discrepancy.

Second, we confirm qualitatively the Peierls stresses of the dislocations between the different lattice orientations as follows. The two dislocations $\left(120^{\circ}\right.$ and $\left.180^{\circ}\right)$ based on the lattice orientation $x=<\overline{1} 10>, y=<111>$ and $z=<11 \overline{2}>$ have higher Peierls stresses than the other dislocations. It can be understood with the density of atoms in the gliding direction. We see in Fig. 13 that the densities vary with the lattice orientations of the slip plane. Therefore, the dislocations built within the same family of lattice orientation having small density have high Peierls stresses. Consequently, the $109.107^{\circ}$ and $130.893^{\circ}$ dislocations have small Peierls stresses compared to the other dislocations.

\section{Conclusion}

In this article, we presented one part of the coupled atomistic discrete dislocation formulation for the three dimensional case. This important part, the so-called core template, deals with dislocations traversing the coupling interface in order to impose a matching boundary condition to the MD. This template is defined by the core atomistic positions which depend on the character angle. These positions can be obtained by modeling a straight mixed dislocation via an atomistic simulation. The detailed setup of such simulations has been extensively described in this paper. Furthermore, based on the extensive modeling results, Peierls stresses have been measured.

As expected, the core size of the modeled straight dislocations varied with the character angle. More specifically, the fault widths between the two partials are decreasing when the character angles increase. This can be understood by considering the variation of the elastic energy. Also, the MD core structures were confirmed by using the variational PN model, which was extended to consider arbitrary angles. The comparison of 
the core structures obtained from both methods showed good agreements. The extended PN method can predict displacement fields for any angle, including these that are difficult to obtain with periodic and finite size MD simulations. Furthermore, the measured Peierls stresses were qualitatively confirmed, and we found that the magnitude of dissociation and the density of atoms in the gliding direction decide the Peierls stress of the dislocation.

As we already mentioned, these results are directly applicable to the CADD3D implementation to provide matching boundary conditions. However, another important component of CADD3D deals with the dynamic motion of dislocations. Moving dislocations partially MD and partially DDD - need to have comparable speeds in order to avoid non-physical spurious forces at the interface. The presented core structures can straightforwardly be employed as a starting point for simulating MD dislocation motion under shear stress and thus to carry out mobility laws for arbitrary mixed angle.

\section{Endnotes}

${ }^{1}$ Swiss national science foundation project conducted by three principal investigators, William Curtin, Jean-François Molinari and Guillaume Anciaux.

${ }^{2}$ Due to plane-strain restrictions, two-dimensional CADD can only handle edge dislocations.

${ }^{3}$ Another method, AtoDis [41] has similar properties, but it has not been shown to be operational in the presence of more than a single dislocation line.

${ }^{4}$ The stacking falut sequence of the dislocation core region $(\mathrm{A} \rightarrow \mathrm{B} \rightarrow \mathrm{A})$ allows us to see the second layer of $A$ atoms. These atoms are not seen in the stacking sequence of perfect FCC crystal systems $(\mathrm{A} \rightarrow \mathrm{B} \rightarrow \mathrm{C} \rightarrow \mathrm{A})$.

Competing interests

The authors declare that they have no competing interests.

Authors' contributions

JC implemented the simulations, and drafted the manuscript. TJ, JFM and GA contributed in the direction of the study and edited the manuscript. All authors read and approved the final manuscript.

\section{Acknowledgments}

This work is supported by the Swiss National Science Foundation (grant no. 200021-140506/1).

Received: 29 December 2014 Accepted: 5 May 2015

Published online: 18 June 2015

\section{References}

1. Ghoniem N, Tong S, Sun L (2000) Parametric dislocation dynamics: A thermodynamics-based approach to investigations of mesoscopic plastic deformation. Phys Rev B 61:913-927

2. Zbib H, Rubia T (2002) A multiscale model of plasticity. Int J Plast 18(9):1133-1163

3. Verdier M, Fivel M, Groma I (1998) Mesoscopic scale simulation of dislocation dynamics in FCC metals: Principles and applications. Modell Simul Mater Sci Eng 6(6):755

4. Kubin L, Canova G, Condat M, Devincre B, Pontikis V, Bréchet Y (1992) Dislocation structures and plastic flow: a 3D simulation. Solid State Phenomena 23(24):455-472

5. Bulatov V, Cai W (2006) Computer simulations of dislocations. Oxford University Press, Oxford, New York. http://opac. inria.fr/record=b1131935

6. Greer J, Weinberger C, Cai W (2008) Comparing the strength of FCC and BCC sub-micrometer pillars: Compression experiments and dislocation dynamics simulations. Mater Sci Eng A 493(1-2):21-25. Mechanical Behavior of Nanostructured Materials, a Symposium Held in Honor of Carl Koch at the \{TMS\} Annual Meeting 2007, Orlando, Florida

7. Martínez E, Marian J, Arsenlis A, Victoria M, Perlado J (2008) Atomistically informed dislocation dynamics in FCC crystals. J Mech Phys Solids 56(3):869-895

8. Fivel M, Robertson C, Canova G, Boulanger L (1998) Three-dimensional modeling of indent-induced plastic zone at a mesoscale. Acta Materialia 46(17):6183-6194

9. Robertson C, Fivel M (1999) A study of the submicron indent-induced plastic deformation. J Mater Res 14:2251-2258

10. Chang H, Fivel M, Rodney D, Verdier M (2010) Multiscale modelling of indentation in FCC metals: From atomic to continuum. Comptes Rendus Physique 11(3-4):285-292. Computational metallurgy and scale transitions Métallurgie numérique et changements d'échelle 
11. Tadmor E, Ortiz M, Phillips R (1996) Quasicontinuum analysis of defects in solids. Philos Mag A 73(6):1529-1563

12. Xiao S, Belytschko T (2004) A bridging domain method for coupling continua with molecular dynamics. Comput Methods Appl Mech Eng 193(17-20):1645-1669. Multiple Scale Methods for Nanoscale Mechanics and Materials

13. Kohlhoff S, Gumbsch P, Fischmeister H (1991) Crack propagation in BCC crystals studied with a combined finite-element and atomistic model. Philos Mag A 64(4):851-878

14. Shilkrot L, Ronald E, Curtin W (2004) Multiscale plasticity modeling: coupled atomistics and discrete dislocation mechanics. J Mech Phys Solids 52(4):755-787

15. Bulatov V, Kaxiras E (1997) Semidiscrete variational peierls framework for dislocation core properties. Phys Rev Lett 78:4221-4224

16. Shiari B, Miller R, Curtin W (2005) Coupled atomistic/discrete dislocation simulations of nanoindentation at finite temperature. J Eng Mater Technol Trans ASME 127(4):358-368

17. Qu S, Shastry V, Curtin W, Miller R (2005) A finite-temperature dynamic coupled atomistic/discrete dislocation method. Modell Simul Mater Sci Eng 13(7):1101-1118

18. Hirth J, Lothe J (1992) Theory of Dislocations. Krieger Publishing Company

19. Junge T (2014) Modelling Plasticity in Nanoscale Contact. PhD thesis, ENAC, Lausanne

20. Mendelev M, Kramer M, Becker C, Asta M (2008) Analysis of semi-empirical interatomic potentials appropriate for simulation of crystalline and liquid Al and Cu. Philos Mag 88(12):1723-1750

21. Burgers J (1940) Geometrical considerations concerning the structural irregularities to be assumed in a crystal. Proc Phys Soc 52(1):23

22. Nabarro F (1947) Dislocations in a simple cubic lattice. Proc Phys Soc 59(2):256

23. Schoeck G (2012) The core structure and peierls potential of dislocations in Al. Mater Sci Eng A 558(0):162-169

24. Hunter A, Beyerlein I, Germann T, Koslowski M (2011) Influence of the stacking fault energy surface on partial dislocations in FCC metals with a three-dimensional phase field dislocations dynamics model. Phys Rev B 84:144108

25. Ngan A (1997) A generalized Peierls-Nabarro model for nonplanar screw dislocation cores. J Mech Phys Solids 45(6):903-921

26. Denoual C (2007) Modeling dislocation by coupling Peierls-Nabarro and element-free Galerkin methods. Comput Methods Appl Mech Eng 196(13-16):1915-1923

27. Shen C, Wang Y (2003) Phase field model of dislocation networks. Acta Materialia 51(9):2595-2610

28. Shen C, Wang Y (2004) Incorporation of $\gamma$-surface to phase field model of dislocations: simulating dislocation dissociation in FCC crystals. Acta Materialia 52(3):683-691

29. Zimmerman J, Gao H, Abraham F (2000) Generalized stacking fault energies for embedded atom FCC metals. Modell Simul Mater Sci Eng 8(2):103

30. Vitek V (1968) Intrinsic stacking faults in body-centred cubic crystals. Philos Mag 18(154):773-786

31. Swygenhoven H, Derlet P, Frøseth A (2004) Stacking fault energies and slip in nanocrystalline metals. Nat Mater 3:399

32. Mishin $Y$, Farkas D, Mehl M, Papaconstantopoulos D (1999) Interatomic potentials for monoatomic metals from experimental data and ab initio calculations. Phys Rev B 59:3393-3407

33. Hunter A, Zhang R, Beyerlein I, Germann T, Koslowski M (2013) Dependence of equilibrium stacking fault width in FCC metals on the $\gamma$-surface. Modell Simul Mater Sci Eng 21(2):025015

34. Kuksin A, Stegailov V, Yanilkin A (2008) Molecular-dynamics simulation of edge-dislocation dynamics in aluminum. Doklady Phys 53(6):287-291

35. Srinivasan S, Liao X, Baskes M, McCabe R, Zhao Y, Zhu Y (2005) Compact and dissociated dislocations in aluminum: implications for deformation. Phys Rev Lett 94:125502

36. Cai W, Bulatov V, Chang J, Li J, Yip S (2004) Dislocation core effects on mobility. In: Nabarro FRN, Hirth J (eds). Dislocations in Solids, vol. 12. North-Holland, Amsterdam

37. Olmsted D, Hardikar K, Phillips R (2001) Lattice resistance and Peierls stress in finite size atomistic dislocation simulations. Modell Simul Mater Sci Eng 9(3):215

38. Shin I, Carter E (2013) Possible origin of the discrepancy in Peierls stresses of FCC metals: First-principles simulations of dislocation mobility in aluminum. Phys Rev B 88:064106

39. Simmons J, Rao S, Dimiduk D (1997) Atomistics simulations of structures and properties of $\frac{1}{2}(110)$ dislocations using three different embedded-atom method potentials fit to $\gamma$-TiAl. Philos Mag A 75(5):1299-1328

40. Cai W (2001) Atomistic and Mesoscale Modeling of Dislocation Mobility. http://dspace.mit.edu/bitstream/handle/ 1721.1/8682/49725239-MIT.pdf

41. Brinckmann S, Mahajan D, Hartmaier A (2012) A scheme to combine molecular dynamics and dislocation dynamics. Modelling Simul Mater Sci Eng 20(4):045001

42. Angelo J, Moody N, Baskes M (1995) Trapping of hydrogen to lattice defects in nickel. Modell Simul Mater Sci Eng 3(3):289

43. Voter A, Chen S (1987) Accurate interatomic potentials for Ni, Al and Ni3Al. Proc MRS Fall Symp 82:175-80

44. Oh D, Johnson R (1988) Simple embedded atom method model for FCC and HCP metals. J Mater Res 3:471-478

45. Hartford J, Sydow B, Wahnstroem G, Lundqvist B (1998) Peierls barriers and stresses for edge dislocations in Pd and Al calculated from first principles. Phys Rev B 58:2487-2496

46. Ercolessi F, Adams J (1994) Interatomic potentials from first-principles calculations: the force-matching method. EPL (Europhys Lett) 26(8):583 